BNL-108514-2015-JA

\title{
Effects of Chemo-Mechanical Polishing on CdZnTe X-Ray and Gamma-Ray Detectors
}

\author{
Stephen U. Egarievwe ${ }^{1,2}$, Anwar Hossain ${ }^{2}$, Ifechukwude O. Okwechime ${ }^{1}$, Rubi \\ $\mathrm{Gul}^{1,2}$, and Ralph B. James ${ }^{2}$ \\ ${ }^{1}$ Nuclear Engineering and Radiological Science Center, Alabama A\&M University, \\ Normal, AL 35762, USA \\ ${ }^{2}$ Department of Nonproliferation and National Security, Brookhaven National Laboratory, \\ Upton, NY 11973, USA
}

Submitted to the Journal of Electronic Materials

June 2015

Nonproliferation and National Security Department Radiation Detector and Research and Development Group

Brookhaven National Laboratory

P.O. Box 5000

Upton, New York 11973

www.bnl.gov

\section{U.S. Department of Energy \\ Defense Nuclear Nonproliferation Research and Development NA-22}

Notice: This manuscript has been co-authored by employees of Brookhaven Science Associates, LLC under Contract No. DE-SC0012704 with the U.S. Department of Energy. The publisher by accepting the manuscript for publication acknowledges that the United States Government retains a non-exclusive, paid-up, irrevocable, world-wide license to publish or reproduce the published form of this manuscript, or allow others to do so, for United States Government purposes. 


\section{DISCLAIMER}

This report was prepared as an account of work sponsored by an agency of the United States Government. Neither the United States Government nor any agency thereof, nor any of their employees, nor any of their contractors, subcontractors, or their employees, makes any warranty, express or implied, or assumes any legal liability or responsibility for the accuracy, completeness, or any third party's use or the results of such use of any information, apparatus, product, or process disclosed, or represents that its use would not infringe privately owned rights. Reference herein to any specific commercial product, process, or service by trade name, trademark, manufacturer, or otherwise, does not necessarily constitute or imply its endorsement, recommendation, or favoring by the United States Government or any agency thereof or its contractors or subcontractors. The views and opinions of authors expressed herein do not necessarily state or reflect those of the United States Government or any agency thereof. 


\title{
Effects of Chemo-Mechanical Polishing on CdZnTe X-Ray and Gamma-Ray Detectors
}

\author{
Stephen U. Egarievwe ${ }^{1,2}$, Anwar Hossain ${ }^{2}$, Ifechukwude O. Okwechime ${ }^{1}$, Rubi Gul ${ }^{1,2}$, and Ralph B. James ${ }^{2}$ \\ ${ }^{1}$ Nuclear Engineering and Radiological Science Center, Alabama A\&M University, Normal, AL 35762, USA \\ ${ }^{2}$ Department of Nonproliferation and National Security, Brookhaven National Laboratory, Upton, NY 11973, USA
}

\begin{abstract}
Mechanically polishing cadmium zinc telluride (CdZnTe) wafers for X-ray and gamma-ray detectors often is inadequate in removing surface defects caused by cutting them from the ingots. Fabrication-induced defects, such as surface roughness, dangling bonds, and nonstoichiometric surfaces, often are reduced through polishing and etching the surface. In our earlier studies of mechanical polishing with alumina-powder, etching with hydrogen bromide in hydrogen peroxide solution, and chemo-mechanical polishing with bromine-methanol-ethylene glycol solution, we found the chemo-mechanical polishing process produced the least surface leakage current. In this research, we focused on using two chemicals to chemo-mechanically polish the CdZnTe wafers after mechanical polishing, viz., bromine-methanol-ethylene glycol (BME) solution, and hydrogen bromide (HBr) in a hydrogen peroxide and ethylene-glycol solution. We used X-ray photoelectron spectroscopy (XPS), current-voltage (I-V) measurements, and Am-241 spectral-response measurements to characterize and compare the effects of each solution. The results show that the HBr-based solution produced lower leakage current than the BME solution. Results from using the same chemo-mechanical polishing solution on two samples confirmed that the surface treatment affects the bulk current. XPS results indicate that the tellurium-oxide to tellurium peaks ratios for the mechanical polishing process were reduced significantly by chemo-mechanical polishing using the BME solution (78.9\% for $\mathrm{Te} \mathrm{d}_{5 / 2} \mathrm{O}_{2}$ and $76.7 \%$ for $\mathrm{Te} 3 \mathrm{~d}_{3 / 2} \mathrm{O}_{2}$ ) compared to the HBr-based solution (27.6\% for $\mathrm{Te} \mathrm{d}_{5 / 2} \mathrm{O}_{2}$ and $35.8 \%$ for $\mathrm{Te}_{3 / 2} \mathrm{O}_{2}$ ). Spectral-response measurements showed that the $59.5-\mathrm{keV}$ peak of Am-241 remained under the same channel number for all three CdZnTe samples. While the BME-based solution gave a better performance of $7.15 \%$ FWHM compared to $7.59 \%$ FWHM for the HBr-based solution, the latter showed a smaller variation in performance of 0.39\% FWHM over seven days compared to 0.69\% for the BME-based solution.
\end{abstract}

Keywords: CdZnTe; chemo-mechanical polishing; leakage current; spectral response; X-ray photoelectron spectroscopy. 


\section{INTRODUCTION}

Cadmium Zinc Telluride (CdZnTe) has found applications in room-temperature X-ray and gamma-ray detections in radiation monitoring, ${ }^{1-4}$ and it has very high potential in nuclear medicine, ${ }^{5}$ medical imaging systems, ${ }^{6}$ astrophysics, ${ }^{7}$ nuclear nonproliferation, and national security. However, its performance still is limited by bulk- and surface-defects. The latter often come from the surface-fabrication processes employed, and they contribute to surface- and bulk-leakage currents that produce electronic noise, which degrades the energy resolution in CdZnTe X-ray and gamma-ray detectors. ${ }^{8-10}$ While bulk leakage current depends on the crystal's quality, the surface leakage current mainly is caused by surface roughness, dangling bonds, and the non-stoichiometric surfaces formed when cutting detector wafers from the as-grown crystal ingots. The surface properties of CdZnTe detectors influence the electric fields inside the wafers, and thus significantly affect the charge transport in radiation detectors. ${ }^{11,12}$ A rough surface increases the leakage current and creates additional trapping centers that adversely affects the detector's performance. ${ }^{13}$ Therefore, it is important to study surface processes and identify methods that produce the best surfaces for an optimum detection performance.

The fabrication-induced defects often can be reduced through surface polishing and etching. The usual practice is to mechanically polish the surfaces of the wafers with silicon-carbide abrasive papers, starting with a low grade of about 600 grits up to a high grade of about 12 grits, so to produce smooth surfaces. Further smoothening is accomplished by fine polishing with 3.0- to about 0.3-micron alumina powder $\left(\mathrm{Al}_{2} \mathrm{O}_{3}\right)$ on a multi-tex pad. To remove residual damages to the mechanical surfaces, the wafer is chemically etched in bromine-methanol- or hydrogen-bromide-based solutions. In previous experiments, our results from atomic force microscopy (AFM) showed that the surface-area roughness of the CdZnTe wafer was reduced from $9.25 \mathrm{~nm}$ root mean square (RMS) for mechanical polishing, to $2.50 \mathrm{~nm}$ RMS after etching in $2 \%$ bromine-methanol solution. ${ }^{13}$ However, chemical etching of CdZnTe wafers was observed to result in a Te-rich surface layer that is subject to oxidation., ${ }^{8,14}$ It also was reported that CdZnTe wafers with gold electrical contacts gave lower surface currents with mechanically polished samples compared to those that were mechanically polished followed by etching in $1 \%$ bromine-methanol solution. ${ }^{8}$

Recently, we have worked on improving the etching process, ${ }^{15,16}$ as well as replacing the chemical etching step in fabricating the device with chemo-mechanical polishing. ${ }^{17,18}$ Since bromine-methanol solution induces surface features that makes them more conductive, ${ }^{8}$ we explored ways to minimize the usage of bromine and also 
find alternative etchants by using 1) a bromine-based passivated etchant where the residual Br is removed from the surfaces to generate nonconductive smooth surfaces, and, 2) non-bromine-based etchants for treating the polished surfaces. $^{15}$ Our results showed that the Am-241 spectral energy resolution of $20 \%$ after only mechanically polishing of the detector was improved to $19 \%$ by using this process with a variable ratio of hydrogen bromide, hydrogen peroxide, and ethylene glycol, and a 23\% energy resolution for only mechanically polishing, the detector was improved to $17 \%$ by using a mix of potassium dichromate, nitric acid, and ethylene glycol solution. ${ }^{15}$ In the present experiments, we compared the effects of using two chemicals to chemo-mechanically polish CdZnTe wafers after mechanical polishing, viz., 1) bromine-methanol-ethylene glycol (BME) solution, and, 2) hydrogen bromide in hydrogen peroxide and ethylene glycol $\left(\mathrm{HBr}+\mathrm{H}_{2} \mathrm{O}_{2}+\mathrm{C}_{2} \mathrm{H}_{6} \mathrm{O}_{2}\right)$ solution.

\section{EXPERIMENTAL PROCEDURES}

\section{Chemo-mechanical Polishing Using BME- and HBr-based Solutions}

Three samples of 6.4 × 6.4 × $2.8 \mathrm{~mm}^{3}$ were sliced from a detector-grade CdZnTe wafer grown by the Bridgman method. All three, A1, A2, and A3, were mechanically polished with 800-grit and 1200-grit siliconcarbide abrasive papers, then were polished in 3.0- and 0.9-micron alumina powder $\left(\mathrm{Al}_{2} \mathrm{O}_{3}\right)$. Samples A2 and A3 were further polished chemo-mechanically using respectively, 1) a bromine-methanol-ethylene glycol (BME) solution, and 2) hydrogen bromide in hydrogen peroxide and ethylene glycol solution. Sample A1 was kept as the control.

We earlier reported the results from a set of experiments, in which we used three CdZnTe samples, each 5 x

$5 \times 2 \mathrm{~mm}^{3}$, to investigate three processes: Process P1: Mechanically polished with a 0.9- $\mathrm{mm}$ alumina powder finishing; Process P2: Mechanically polished with a 0.9- $\mu \mathrm{m}$ alumina powder and etched with hydrogen bromide in hydrogen peroxide solution for 2 minutes; and, Process P3: Mechanically polished with a 0.9- $\mu$ m alumina powder and chemo-mechanically polished with bromine-methanol-ethylene glycol solution. ${ }^{17}$ Results from the images of the surfaces are presented in this paper.

We undertook an X-ray photoelectron spectroscopy (XPS) experiment using a RHK Technology UHV 7500 system in an ultrahigh-vacuum setup at a pressure below 8 x $10^{-10} \mathrm{~Pa}$ to scan for peaks of cadmium (Cd), tellurium (Te), and tellurium oxide $\left(\mathrm{TeO}_{2}\right)$ on the surfaces of the samples. The XPS chamber was equipped with a 
dual $\mathrm{Al} / \mathrm{Mg} \mathrm{X}$-ray gun and a source from an argon-ion sputtering system. The measurements were acquired through the aluminum X-ray source immediately after polishing each surface.

To measure the bulk leakage current, gold electrical contacts were deposited on the centers of the two opposite planar surfaces of each CdZnTe wafer using a $5 \%$ gold chloride $\left(\mathrm{AuCl}_{3}\right)$ solution that we placed on the detectors' surface using a special pipette. In our technique, it is easy to prevent the $\mathrm{AuCl}_{3}$ solution from spilling over on to other surfaces. The gold contact is formed within 15 to $30 \mathrm{~s}$, after which the excess solution in the $\mathrm{AuCl}_{3}$ droplet is removed in two steps. First, a felt paper is used to absorb the excess $\mathrm{AuCl}_{3}$ solution without touching the detector's surface. Immediately afterwards, we dipped the detector into deionized water, and then quickly blowdried it with pressurized nitrogen gas. The current-voltage (I-V) measurements were acquired using a customized current-voltage probe in a metal box coupled to a Keithley Picoammeter/Voltage Source. The process for measuring surface leakage current differs in that two parallel strips of gold contact are deposited on the same planar surface of the detector using a lithographic technique.

To assess the performances of the detectors after each of the surface polishing techniques, we measured the spectral responses of the three samples for Am-241 at an applied voltage of $200 \mathrm{~V}$. In these measurements, a standard eV Products brass holder was used to secure the detector against a beryllium window with a gold-plated spring contact. The brass holder was connected to a multi-channel analyzer (MCA) through a pre-amplifier and a shaping amplifier. The detector's response signal generated from irradiation from a sealed Am-241 gamma-ray source was recorded through the MCA, and stored for processing and analysis.

\section{Chemo-Mechanical Polishing and Bulk Leakage Current}

During bulk I-V measurements, we observed that the bulk leakage current changes as a result of the chemomechanical polishing process. In principle, it is expected that surface treatment would only affect the surface leakage current and not both surface and bulk leakage currents. In order to confirm that the change of the bulk leakage current comes from the chemo-mechanical polishing process rather than the CdZnTe samples themselves, we performed further experiments on two wafers (samples A4 and A5) sliced from the same region of the ingot.

First, the two detector grade CdZnTe samples A4 and A5 were mechanically polished with 800-grit and 1200-grit silicon-carbide abrasive papers, followed by fine polishing in 3.0- and 0.9-micron alumina powder. Next, sample A4 was kept as control and sample A5 was chemo-mechanically polished using bromine-methanol-ethylene 
glycol solution. Gold contacts were deposited on the opposite planer surfaces of each sample. Bulk I-V measurements were then carried out on samples A4 and A5 using a Keithley Picoammeter/Voltage Source. Next, detector's response signals generated from irradiation from a sealed Am-241 gamma-ray source was recorded for both samples. We then striped the gold contacts from the two wafers and repeated the experiments with the two samples switched: sample A5 now serve as control and sample A4 was chemo-mechanically polished using bromine-methanol-ethylene glycol solution after mechanical polishing of the sample.

\section{RESULTS AND DISCUSSION}

\section{Images of Polished, Etched and Chemo-Mechanically Polished Surfaces}

Figure 1 shows the images of polished, etched, and chemo-mechanically polished surfaces using an optical microscope fitted with a CCD camera for the three processes: Mechanically polished with a $0.9-\mu \mathrm{m}$ alumina powder finishing (P1), mechanically polished with a $0.9-\mu \mathrm{m}$ alumina powder and etched with hydrogen bromide in hydrogen peroxide solution for 2 minutes (P2), and mechanically polished with a $0.9-\mu \mathrm{m}$ alumina powder and chemo-mechanically polished with bromine-methanol-ethylene glycol solution (P3). The surface that was smoothest was the chemo-mechanically polished one. Atomic force microscopy (AFM) of the three surfaces showed

roughness of $3.76 \mathrm{~nm}, 37.35 \mathrm{~nm}$, and $1.97 \mathrm{~nm}$ for P1, P2 and P3 respectively. ${ }^{17}$ The roughness of the surfaces plays an important role in the amount of leakage current and charges that are trapped. The rougher surfaces, enhance more leakage current that goes into the detector medium and also create additional charge-carrier trapping centers that result in degrading energy resolution and large low-energy tail of photopeaks. ${ }^{8,15}$ While mechanical polishing removes defects mostly introduced during cutting and dicing of the wafers, it also create new defects on the polished surface that contribute to the trapping of charge carriers. ${ }^{15}$ Increased surface roughness leads to more carriers contributing to the dark current and thus lowers the signal-to-noise ratio, which in turn lowers the spectral resolution of the detector.

\section{Surface and Bulk Leakage Currents}

Surface leakage currents were measured processes P1, P2, and P3. The results, shown in Fig. 2, indicate that chemo-mechanical polishing with bromine-methanol-ethylene glycol solution was the best method of the three 
for reducing surface leakage current; currents at 20 volts were $1.44 \mathrm{nA}, 0.23 \mathrm{nA}$, and $0.03 \mathrm{nA}$ for P1, P2, and P3 respectively. Unlike the increase in surface current caused by etching with bromine methanol solution as shown in Fig. 2, and similar to reports by others, ${ }^{8,19}$ we observed a decrease in the surface current after using chemomechanical polishing with a bromine-methanol-ethylene glycol solution. Zha et al. studied the effect of etching with bromine methanol solution in more detail. ${ }^{19,20}$ In our experiment, we found that chemo-mechanical polishing process was the most effective in reducing the surface leakage current. Therefore, in addition to the surface roughness, the surface species could also affect the amount of surface leakage current.

Measurements of the bulk leakage currents were made to compare two chemical solutions for the chemomechanical polishing process. Bulk voltage-current measurements were made for samples A1, A2 and A3, corresponding respectively to mechanically polishing in 0.9-micron alumina powder, chemo-mechanically polishing in bromine-methanol-ethylene glycol solution, and chemo-mechanically polishing in hydrogen bromide in hydrogen peroxide and ethylene glycol solution. The resistivity of each of the CdZnTe samples is of the order of $10^{10} \Omega$-cm. As shown in Fig. 3, the hydrogen bromide-based solution produced lower bulk leakage currents compared to the bromine-methanol-ethylene glycol solution. Since these three samples may have different bulk properties, we further used the same chemo-mechanical polishing solution (bromine-methanol-ethylene glycol) on two samples (A4 and A5) sliced from the same region of the CdZnTe ingot.

While it is expected from principles that surface treatment would only affect the surface leakage current and not bulk resistivity, our experimental results showed changes to the bulk leakage current. The results of treating the two CdZnTe samples A4 and A5 with the same chemo-mechanical polishing solution (Figs. 4 and 5) showed that the experiment is repeatable. The bulk I-V curves of samples A5 chemo-mechanically polished using brominemethanol-ethylene glycol while sample A4 serving as control is shown in Fig. 4. Similar results are shown in Fig. 5 with the two samples switched: sample A5 (mechanical polishing only) serving as control and sample A (mechanical polished followed by chemo-mechanically polished). The results in both Figs. 4 and 5 confirmed that the change of the bulk leakage current comes from the chemo-mechanical polishing process rather than the CdZnTe samples themselves. This result could be attributed to the effect of the surface treatment (chemo-mechanical polishing) on the electric field created in the bulk of the CdZnTe wafers. The effects of surface roughness and material-composition on charge trapping influences the amount of charges collected at the electrodes, hence the change in the recorded bulk leakage current. 


\section{Surface Composition Analysis}

The dominant species on various polished surfaces were determined by XPS experiments. The results are shown in Figs. 6 and 7. Figure 6 shows the XPS spectra of the Te $3 d$ bands of the CdZnTe samples: A1 (mechanically polished in 0.9-micron alumina powder); A2 (chemo-mechanically polishing in bromine-methanolethylene glycol solution); and A3 (chemo-mechanically polishing in hydrogen bromide in hydrogen peroxide and ethylene glycol solution). The Te $3 d$ doublet corresponding to the Te elemental state was found on the three samples at binding energies of approximately $572 \mathrm{eV}$ and $583 \mathrm{eV}$. Two additional peaks appeared at higher binding energies of approximately $576 \mathrm{eV}$ and $587 \mathrm{eV}$, close to the $\mathrm{Te} 3 \mathrm{~d}$ doublet, that reflects the generation of the $\mathrm{TeO}_{2}$ state. The tellurium-oxide peaks $\mathrm{Te} 3 \mathrm{~d}_{5 / 2} \mathrm{O}_{2}$ and $\mathrm{Te} 3 \mathrm{~d}_{3 / 2} \mathrm{O}_{2}$ are prominent on the CdZnTe sample that was mechanically polished in 0.9-micron alumina powder (A1) and the sample that was chemo-mechanically polished in hydrogen bromide in hydrogen peroxide and ethylene glycol solution (A3). As shown in Tables I and II, these tellurium-oxide peaks were lower in the CdZnTe sample that was chemo-mechanically polishing in brominemethanol-ethylene glycol solution (A2). Each peak-height in Tables I and II was measured from the base of the peak and not from zero intensity. The tellurium-oxide to tellurium peaks ratios for the mechanical polishing process were reduced significantly by chemo-mechanical polishing using the BME solution (78.9\% for $\mathrm{Te}^{2} \mathrm{~d}_{5 / 2} \mathrm{O}_{2}$ and $76.7 \%$ for Te3d $\mathrm{d}_{3 / 2} \mathrm{O}_{2}$ ) compared to the HBr-based solution (27.6\% for $\mathrm{Te}_{5 / 2} \mathrm{O}_{2}$ and $35.8 \%$ for $\mathrm{Te}_{3} \mathrm{~d}_{3 / 2} \mathrm{O}_{2}$ ). Thus, the latter treatment is more effective in limiting the production of $\mathrm{TeO}_{2}$ than was the $\mathrm{HBr}$-based solution. The XPS spectra in Fig. 7 revealed prominent $\mathrm{Cd} 3 \mathrm{~d}_{5 / 2}$ and $\mathrm{Cd} 3 \mathrm{~d}_{3 / 2}$ peaks at about $407 \mathrm{eV}$ and $412 \mathrm{eV}$, corresponding with the elemental peak of cadmium. The Cd peaks remained fairly stable for the three samples, thus indicating no significant changes in the Cd peaks were caused by each of the chemo-mechanical polishing solutions.

\section{Detectors’ Performance}

The Am-241 spectral responses were recorded for the CdZnTe samples that were mechanical polished with 0.9-micron alumina powder (A1), chemo-mechanically polished with bromine-methanol-ethylene glycol solution (A2), and chemo-mechanically polished with hydrogen bromide in hydrogen peroxide and ethylene glycol solution (A3). Figure 8 shows these spectra, displaying the 59.5-keV peak of Am-241. The 59.5-keV peak was stable under the same channel for all three samples, but the three samples have slight difference in their spectral performance as 
evidenced by the full-width-at-half-maximum (FWHM) values in Fig. 8. A calculation of the spectral response using the nearest whole-channel numbers gives a 7.5\% FWHM for the 59.5-keV peak of Am-241 for each of the samples. Thus, it is necessary to more precisely calculate the FWHM by extrapolating the fraction of channel numbers that correspond exactly to the FWHM. This approach is the correct one since the channel numbers correspond to energies. After obtaining the counts corresponding to half of the peak count, the FWHM channels then were extrapolated from the nearest recorded counts below and above them, and their corresponding channel numbers. The result gives 7.67\% FWHM for mechanical polishing, 7.15\% FWHM for BME-based chemomechanical polishing, and 7.59\% FWHM for HBr-based chemo-mechanical polishing.

The results of monitoring the Am-241 spectral response over seven days for CdZnTe samples chemomechanically polished in BME-based solution and HBr-based solution are shown, respectively, in Figs. 9 and 10. The 59.5-keV peak of Am-241 was stable under one channel number over seven days for both chemicals. The variation in the FWHM for the BME-based chemo-mechanically polished CdZnTe detectors over the seven days is $0.69 \%$, with a continuous decrease in performance $(6.72 \%, 7.02 \%, 7.17 \%$, and $7.41 \%$ for days 1,3 , 4 , and 7 , respectively). In contrast, the variation in the FWHM for the HBr-based chemo-mechanically polished CdZnTe detectors was $0.39 \%$ and improved each day, increasing after day 4 . These results show that measurements of spectral response over long periods are need to better understand the long-term effects of surface chemo-mechanical polishing and chemical treatments on the performance of CdZnTe detectors.

\section{CONCLUSIONS}

We detailed the effects of mechanical polishing, chemical etching, and chemo-mechanical polishing on the performance of CdZnTe nuclear detectors. Then, we compared the outcomes for two chemo-chemical polishing solutions. The increase in surface current caused by etching with bromine methanol solution as reported in the literature, ${ }^{8,19}$ was greatly reduced in our experiments by lowering the effect of bromine by using a brominemethanol-ethylene glycol solution - this yielded a current of $0.23 \mathrm{nA}$ compared to mechanical polishing with 1.44 nA for the applied voltage of $20 \mathrm{~V}$. Chemo-mechanical polishing with bromine-methanol-ethylene glycol solution reduced the surface current further to $0.02 \mathrm{nA}$ at $20 \mathrm{~V}$, thus proving that chemo-mechanical polishing is the most effective among the solutions studied in reducing the surface leakage current. Comparing the chemo-chemical polishing solutions showed that hydrogen bromide-based solution produced lower bulk leakage currents than did 
bromine-methanol-ethylene glycol solution. However, our XPS spectra records revealed that chemo-mechanical polishing with bromine-methanol-ethylene glycol solution is more effective in limiting the production of $\mathrm{TeO}_{2}$ than is that with the HBr-based solution. Since the three samples for the measurement may have different bulk properties, we further used the same chemo-mechanical polishing solution (bromine-methanol-ethylene glycol) on two samples (A4 and A5) sliced from the same region of the CdZnTe ingot, and the results confirmed that the surface treatment affects the bulk current. This result could be attributed to the effects of surface roughness and material-composition on charge-trapping which in turn influences the amount of charges collected at the electrodes, hence the change in the recorded bulk leakage current. Spectral-response measurements showed that the 59.5-keV peak of Am-241 remained under the same channel number for CdZnTe samples that were mechanically polished, chemo-mechanical polished with hydrogen bromide-based solution, and chemo-mechanically polished with bromine-methanol-ethylene glycol solution. While the BME-based solution gave a better performance of 7.15\% FWHM compared to $7.59 \%$ FWHM for the HBr-based solution, the latter treatment resulted in a smaller variation in performance of $0.39 \%$ FWHM over seven days compared to $0.69 \%$ for the BME-based solution.

\section{ACKNOWLEDGEMENTS}

This work has been supported by the U.S. Department of Homeland Security, Domestic Nuclear Detection Office, under competitively awarded contract/IAA award number 2012-DN-077-ARI065-03. Alabama A\&M University researchers were also supported by the U.S. Nuclear Regulatory Commission through award number NRC-27-10514, and BNL scientists received support from the U.S. Department of Energy Office of Defense Nuclear Nonproliferation R\&D. These supports do not constitute an expressed or implied endorsement by the U.S. Government.

\section{REFERENCES}

1 L. Verger, J. P. Bonnefoy, F. Glasser, and P. Ouvrier-Buffet, Journal of Electronic Materials 26-6, 738 (1997).

2 R. B. James, T. E. Schlesinger, J. C. Lund, and M. Schieber, Semiconductors for room temperature nuclear detector applications, Academic Press, San Diego, vol. 43 (1995). 
3 T. E. Schlesinger, J. E. Toney, H. Yoon, E. Y. Lee, B. A. Brunett, L. Franks, and R. B. James, Material Science and Engineering 32, 103 (2001).

${ }^{4}$ S. Del Sordo, L. Abbene, E. Caroli, A. M. Mancini, A. Zappettini and P. Ubertini, Sensors 9, 3491 (2009).

${ }^{5}$ C. Scheiber, Nuclear Instruments and Methods in Physics Research A 448, 513 (2000).

${ }^{6}$ L. Verger, M. Boitel, M. C. Gentet, R. Hamelin, C. Mestais, F. Mongellaz, J. Rustique, G. Sanchez, Nuclear Instruments and Methods in Physics Research A 458, 297 (2001).

7 O. Limousin, Nuclear Instruments and Methods in Physics Research A 504, 24 (2003).

${ }^{8}$ M. C. Duff, D. B. Hunter, A. Burger, M. Groza, V. Buliga, and D. R. Black, Applied Surface Science 254, 2889 (2008).

${ }^{9}$ T. H. Prettyman, F. P. Ameduri, A. Burger, J. C. Gregory, M. A. Hoffbauer, P.R. Majerus, D. B. Reisenfeld, S. A. Soldner, and C. Szeles, Proc. SPIE 4507, 25 (2001).

${ }^{10}$ A. Hossain, A. E. Bolotnikov, G. S. Camarda, Y. Cui, S. Babalola, A. Burger, and R. B. James, Journal of Electronic Materials 38, 1356 (2008).

${ }^{11}$ T. H. Prettyman, F. P. Ameduri, A. Burger, J. C. Gregory, M. A. Hoffbauer, P. R. Majerus, D. B. Reisenfeld, S. A. Soldner and C. Szeles, Proc. SPIE 4507, 25 (2001).

${ }^{12}$ T. H. Prettyman, M. A. Hoffbauer, J. A, Rennie, S. Cook, J. C. Gregory, M. A. George, P. N. Luke, M. Amman, S. A. Soldner and J. R. Earnhart, Nucl. Instr. and Meth. A 422, 179 (1999).

${ }^{13}$ A. Hossain, S. Babalola, A. E. Bolotnikov, G. S. Camarda, Y. Cui, G. Yang, M. Guo, D. Kochanowska, A. Mycielski, A. Burger and R. B. James, Proc. of SPIE 7079, 70791E-1 (2008).

${ }^{14}$ K. Chattopadhyay, M. Hayes, J. O. Ndap, A. Burger, M. J. Lu, H. G. McWhinney, T. Grady and R. B. James, Journal of Electronic Materials 29, No. 6, 708 (2000).

${ }^{15}$ A. Hossain, A. E. Bolotnikov, G. S. Camarda, Y. Cui, D. Jones, J. Hall, K. H. Kim, J. Mwathi, X. Tong, G. Yang and R. B. James, Journal of Electronic Materials 43, No. 8, 2771 (2014).

${ }^{16}$ A. Hossain, A. Dowdy, A. E. Bolotnikov, G. S. Camarda, Y. Cui, U. N. Roy, R. Tappero, X. Tong, G. Yang and R. B. James, Journal of Electronic Materials 43, No. 8, 2941 (2014).

${ }^{17}$ D. E. Jones, S. U. Egarievwe, A. Hossain, I. O. Okwechime, M. L. Drabo, J. Hall, A. L. Adams, S. O. Babalola, G. S. Camarda, A. E. Bolotnikov, W. Chan and R. B. James, IEEE Records of Nuclear Science Symposium and Medical Imaging Conference, 4124 (2012). 
${ }^{18}$ I. O. Okwechime, S. U. Egarievwe, A. Hossain, Z. M Hales, A. A. Egarievwe and R. B. James, Proc. SPIE 9213, 92130Y (2014).

${ }^{19}$ G. Zha, W. Jie, T. Tan and X. Wang, Nuclear Instruments and Methods in Physics Research A 566, 495 (2006).

${ }^{20}$ G. Zha, W. Jie, T. Tan and P. Li, Applied Surface Science 253, 3476 (2007). 


\section{TABLES}

Table I. XPS spectral intensities - comparison of reduction in $\mathrm{Te}_{3 / 2} \mathrm{O}_{2}$ to $\mathrm{Te} 3 \mathrm{~d}_{3 / 2}$ peaks height ratio by chemomechanical polishing of CdZnTe surfaces using BME and HBr-based solutions.

\begin{tabular}{lccc}
\hline Surface Polishing & $\mathrm{Te}_{3 / 2}$ Peaks & $\mathrm{Te}_{3 \mathrm{~d}_{3 / 2} \mathrm{O}_{2} \text { Peaks }}$ & $\mathrm{Te} \mathrm{d}_{3 / 2} \mathrm{O}_{2} / \mathrm{Te} \mathrm{d}_{3 / 2}$ \\
& Height (a.u.) & Height (a.u.) & Peaks Height Ratio \\
\hline Mechanical & 10500 & 4500 & 0.53 \\
BME Chemo-mechanical & 15000 & 1500 & 0.10 \\
HBr Chemo-mechanical & 20000 & 5500 & 0.28 \\
\hline
\end{tabular}

Table II. XPS spectral intensities - comparison of reduction in $\mathrm{Te} \mathrm{d}_{5 / 2} \mathrm{O}_{2}$ to $\mathrm{Te} 3 \mathrm{~d}_{5 / 2}$ peaks height ratio by chemomechanical polishing of CdZnTe surfaces using BME and HBr-based solutions.

\begin{tabular}{lccc}
\hline Surface Polishing & $\begin{array}{c}\mathrm{Te} \mathrm{d}_{5 / 2} \text { Peaks } \\
\text { Height (a.u.) }\end{array}$ & $\begin{array}{c}\mathrm{Te}_{5 / 2} \mathrm{O}_{2} \text { Peaks } \\
\text { Height (a.u.) }\end{array}$ & $\begin{array}{c}\mathrm{Te}_{5 / 2} \mathrm{O}_{2} / \mathrm{Te} 3 \mathrm{~d}_{5 / 2} \\
\text { Peaks Height Ratio }\end{array}$ \\
\hline Mechanical & 15900 & 7100 & 0.45 \\
BME Chemo-mechanical & 23300 & 2200 & 0.09 \\
HBr Chemo-mechanical & 32500 & 10500 & 0.32 \\
\hline
\end{tabular}


FIGURES

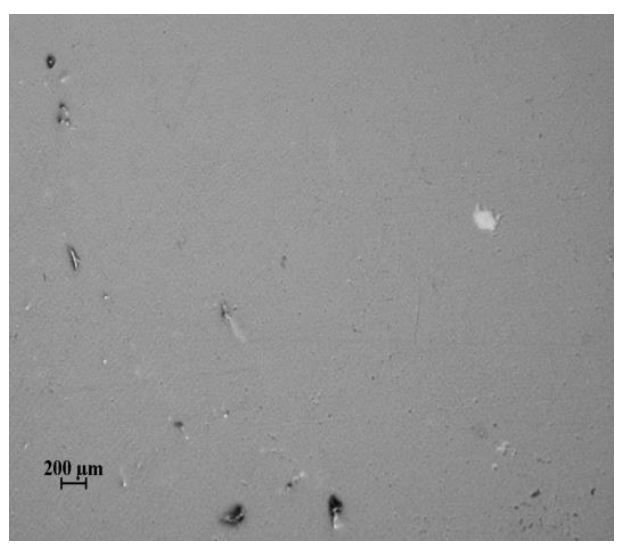

(a)

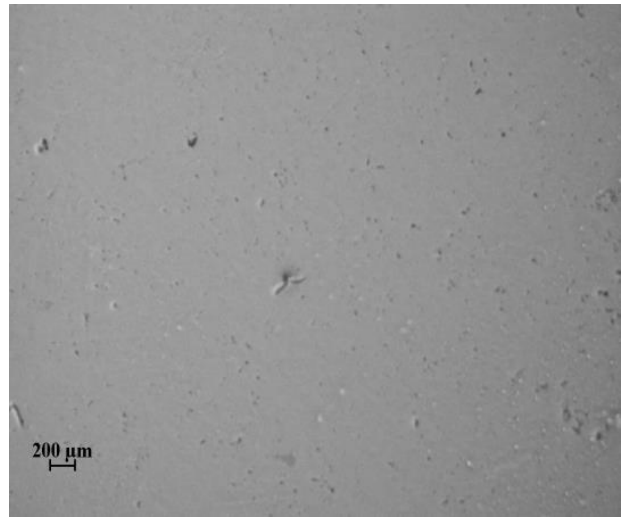

(b)

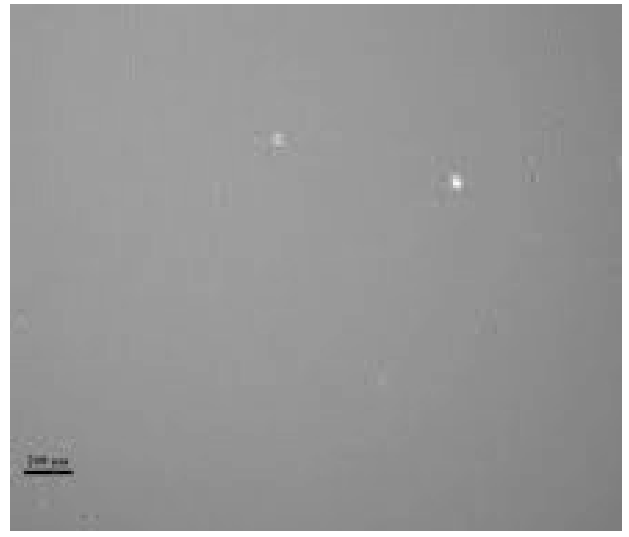

(c)

Fig. 1. Optical images of polished, etched, and chemo-mechanically polished CdZnTe wafers using an optical microscope fitted with a CCD camera. (a) Mechanically polished with a $0.9-\mu \mathrm{m}$ alumina powder finishing. (b) Mechanically polished with a 0.9- $\mu \mathrm{m}$ alumina powder and etched with hydrogen bromide in hydrogen peroxide solution for 2 minutes. (c) Mechanically polished with a 0.9- $\mu \mathrm{m}$ alumina powder and chemo-mechanically polished with bromine-methanol-ethylene glycol solution. 


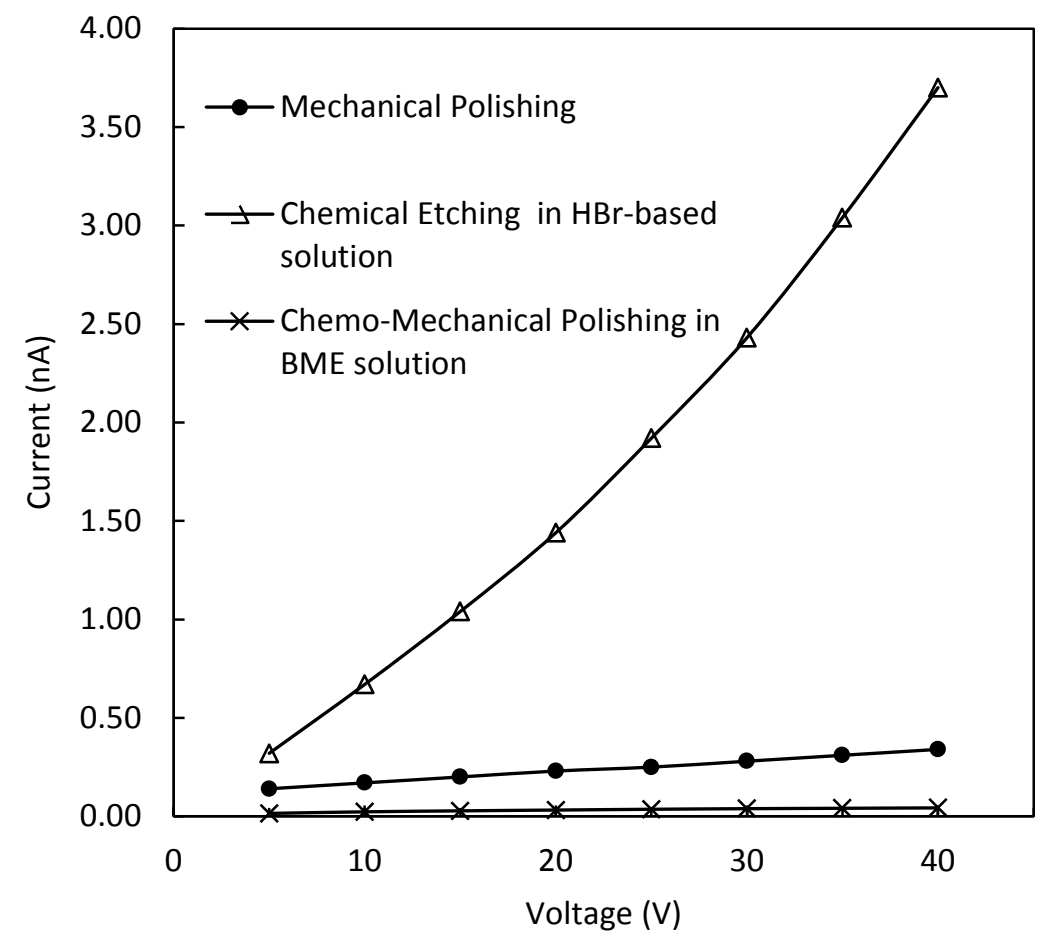

Fig. 2. Surface leakage current for CdZnTe wafers mechanical polished (with a $0.9-\mu \mathrm{m}$ alumina powder finishing), chemical etched (with hydrogen bromide in hydrogen peroxide solution for 2 minutes), and chemo-mechanically polished (bromine-methanol-ethylene glycol solution).

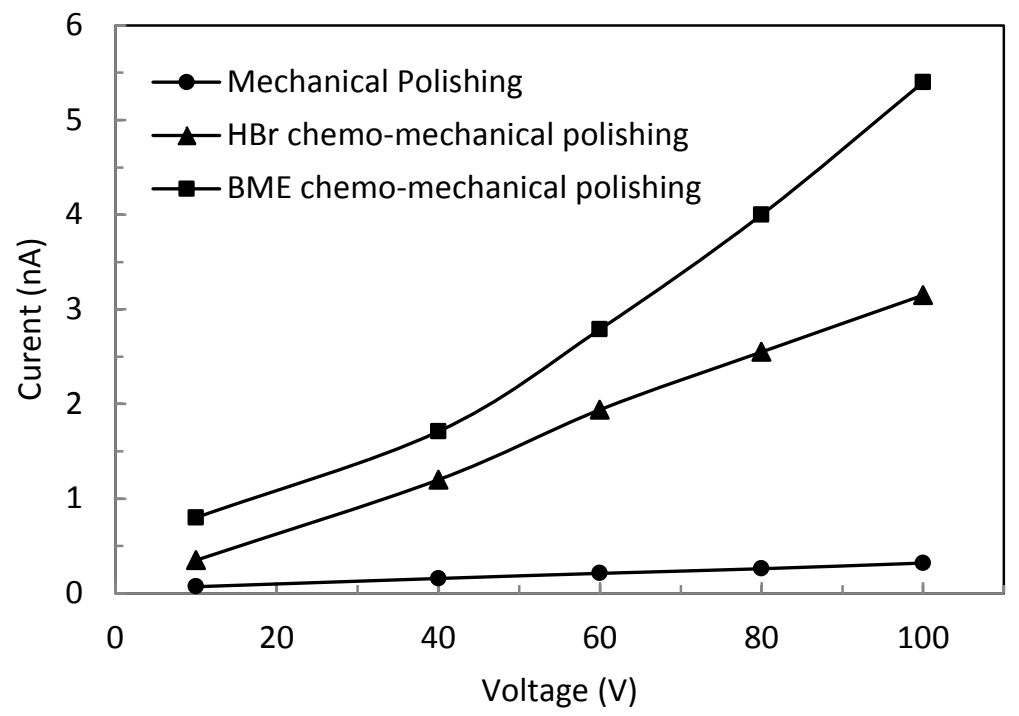

Fig. 3. Bulk voltage-current measurements for CdZnTe wafers mechanically polished in 0.9-micron alumina powder, chemo-mechanically polished in bromine-methanol-ethylene glycol solution, and chemo-mechanically polished in hydrogen bromide in hydrogen peroxide and ethylene glycol solution. 


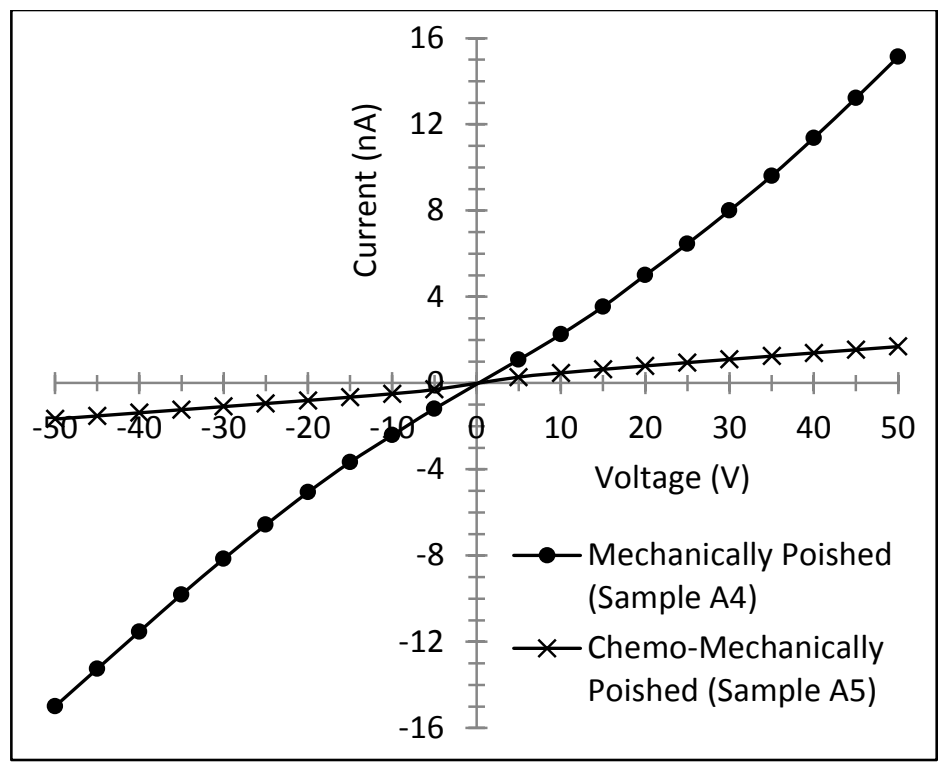

Fig. 4. Bulk voltage-current measurements for CdZnTe Sample A4 as control sample (mechanically polished only) and Sample A5 (mechanically polished followed with chemo-mechanically polished in bromine-methanol-ethylene glycol solution).

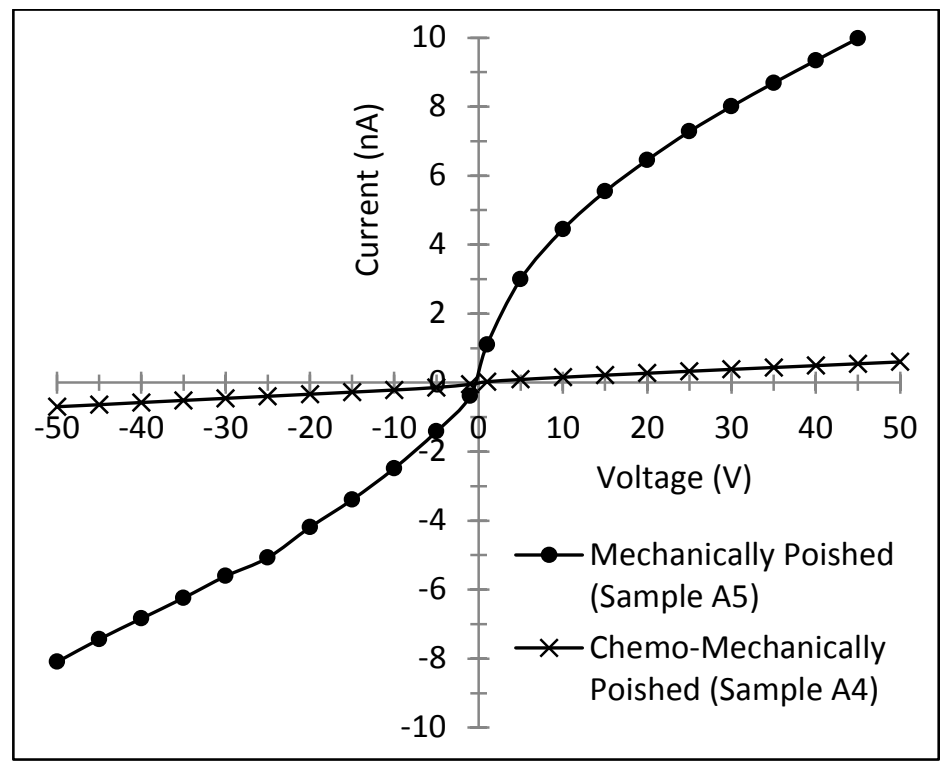

Fig. 5. Bulk voltage-current measurements for CdZnTe Sample A5 as control sample (mechanically polished only) and Sample A4 (mechanically polished followed with chemo-mechanically polished in bromine-methanol-ethylene glycol solution). 


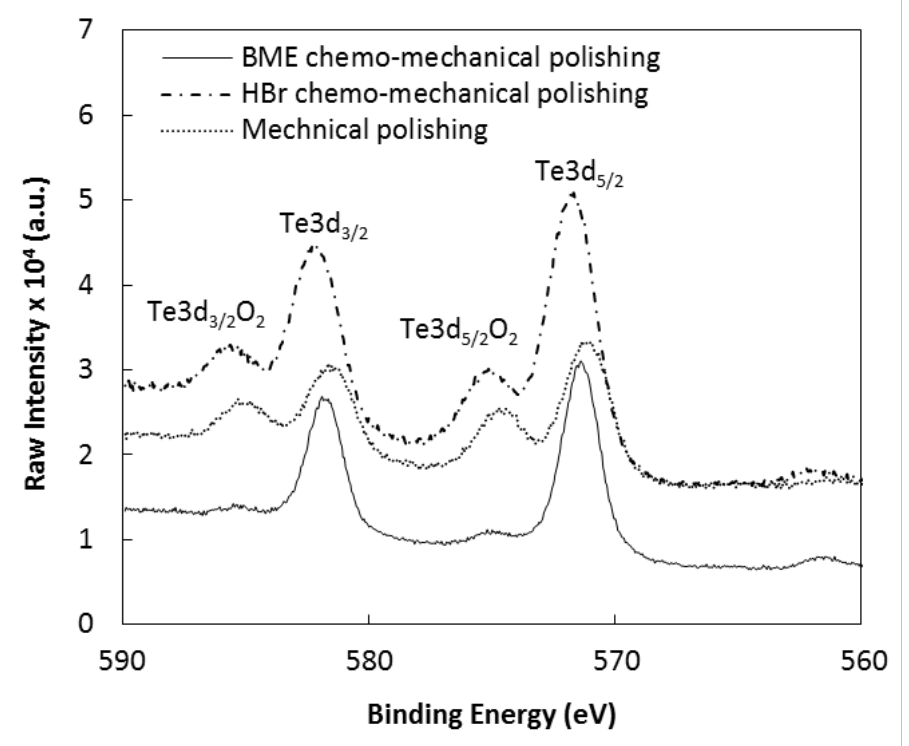

Fig. 6. XPS spectra showing the $\mathrm{TeO}_{\mathrm{x}}$ peaks for $\mathrm{CdZnTe}$ wafers mechanically polished in 0.9-micron alumina powder, chemo-mechanically polishing in bromine-methanol-ethylene glycol solution, and chemo-mechanically polished in hydrogen bromide in hydrogen peroxide and ethylene glycol solution.

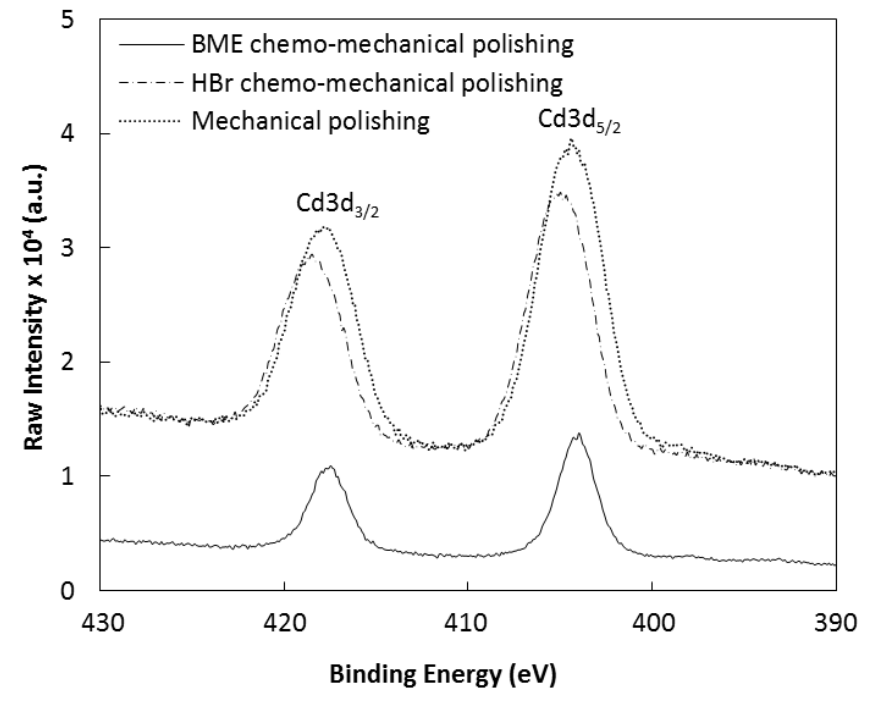

Fig. 7. XPS spectra showing $C d 3 d_{3 / 2}$ and $C d 3 d_{5 / 2}$ peaks for CdZnTe wafers mechanically polished in 0.9-micron alumina powder, chemo-mechanically polished in bromine-methanol-ethylene glycol solution, and chemomechanically polished in hydrogen bromide in hydrogen peroxide and ethylene glycol solution. 


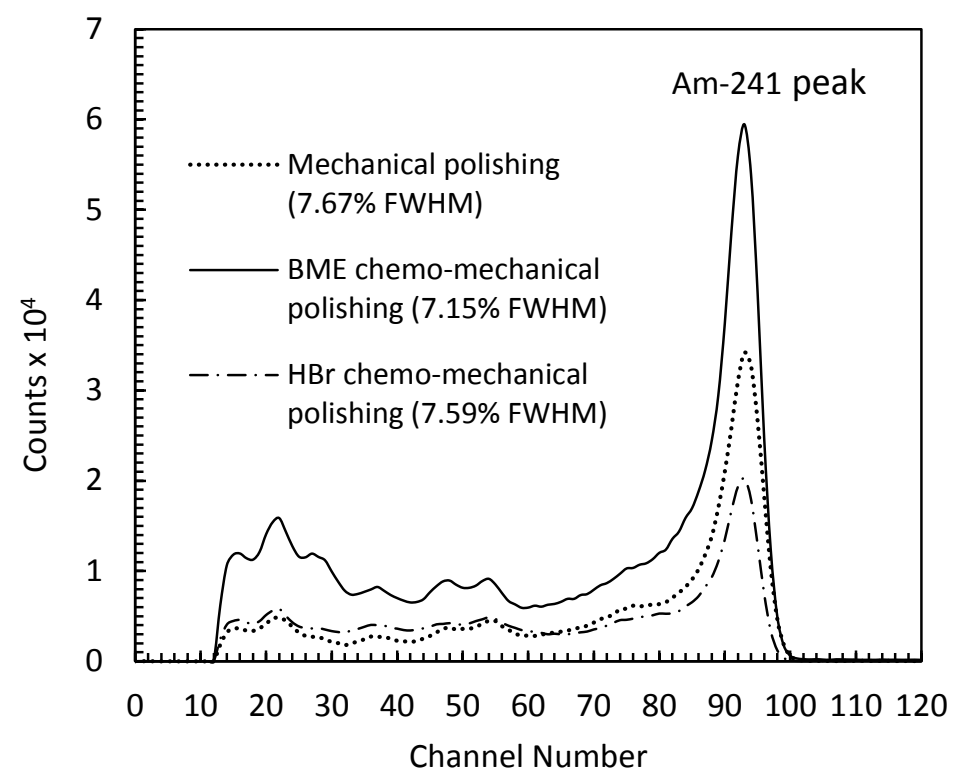

Fig. 8. Am-241 spectral responses of CdZnTe detectors mechanically polished in 0.9-micron alumina powder, chemo-mechanically polished in bromine-methanol-ethylene glycol solution, and chemo-mechanically polished in hydrogen bromide in hydrogen peroxide and ethylene glycol solution.

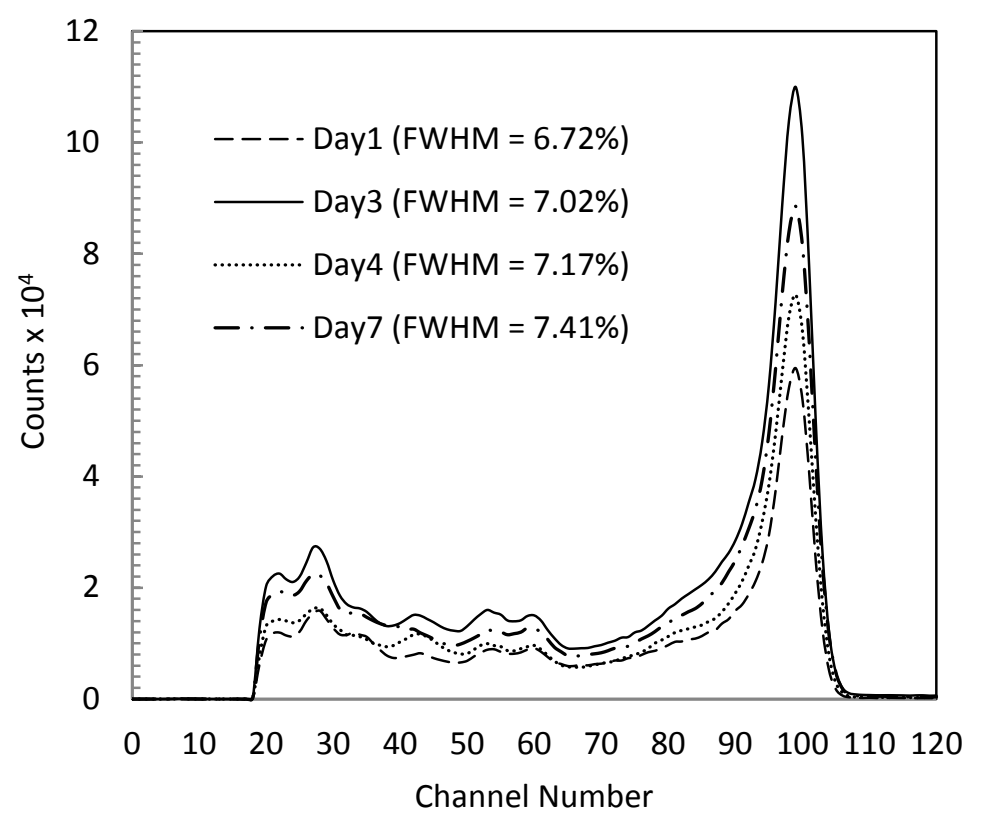

Fig. 9. Am-241 spectral responses over seven days for a CdZnTe detector chemo-mechanically polished in bromine-methanol-ethylene glycol solution. The variation in FWHM over seven days is $0.69 \%$ with a continuous decrease in performance. 


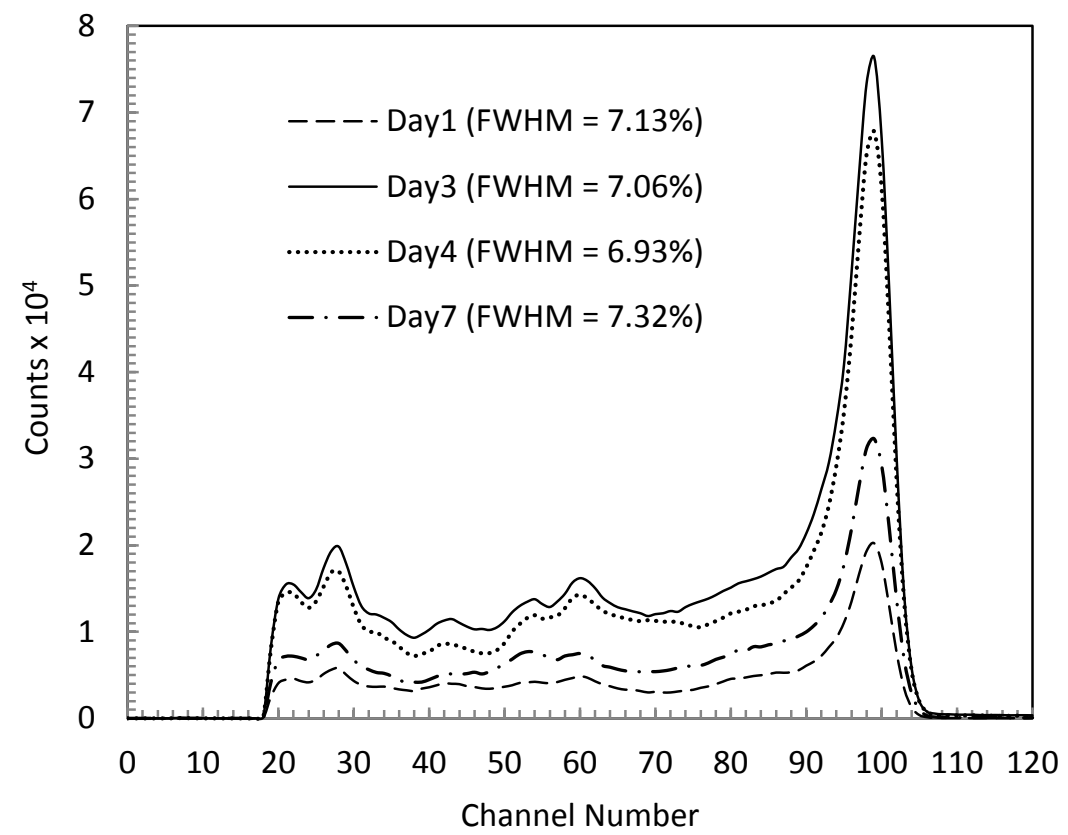

Fig. 10. Am-241 spectral responses over seven days for a CdZnTe detector chemo-mechanically polished in hydrogen bromide in hydrogen peroxide and ethylene-glycol solution. The variation in FWHM is $0.39 \%$ over seven days. The performance improved each day and increased after day 4 . 